\title{
Sublingual sufentanil for postoperative pain relief: first clinical experiences
}

This article was published in the following Dove Press journal: Journal of Pain Research

\author{
Fleur Meijer' \\ Petra Cornelissen ${ }^{2}$ \\ Corina $\mathrm{Sie}^{3}$ \\ Michel Wagemans ${ }^{4}$ \\ Anja Mars ${ }^{5}$ \\ Tiny Hobma ${ }^{1,6}$ \\ Marieke Niesters' \\ Albert Dahan' \\ J Seppe Koopman ${ }^{6}$ \\ Monique AH Steegers ${ }^{2}$ \\ 'Department of Anesthesiology, \\ Leiden University Medical Centre, \\ Leiden, the Netherlands; ${ }^{2}$ Department \\ of Anesthesiology Pain and Palliative \\ Medicine, Radboud University Medical \\ Centre, Nijmegen, the Netherlands; \\ ${ }^{3}$ Department of Anesthesiology, \\ Erasmus Medical Centre, Rotterdam, \\ the Netherlands; ${ }^{4}$ Department of \\ Anesthesiology and Pain Medicine, \\ Reinier de Graaf Hospital, Delft, \\ the Netherlands; ${ }^{5}$ Department of \\ Anesthesiology and Acute Pain \\ Service, Alrijne Hospital, Leiderdorp, \\ the Netherlands; ${ }^{6}$ Department of \\ Anesthesiology, Maasstad Hospital, \\ Rotterdam, the Netherlands
}

Background: The sublingual sufentanil tablet system (SSTS) is a novel hand-held patientcontrolled analgesia device developed for treatment of moderate-to-severe postoperative pain. Here we present the first results of its clinical use.

Methods: Adult patients undergoing major surgery in five hospitals in the Netherlands received the SSTS for postoperative pain relief as part of multimodal pain management that further included paracetamol and a nonsteroidal anti-inflammatory drug (NSAID). The following variables were collected: postoperative pain scores using the 11-point numerical rating score (NRS) at rest, number of tablets used, occurrence of nausea, and patient satisfaction scores.

Results: We included 280 patients in the study; the majority underwent laparoscopic abdominal (49\%) or orthopedic (knee replacement) surgery (34\%). The median NRS was 3.5 (interquartile range 2.3-4.0) on the day of surgery, $3.3(2.3-4.0)$ on the first postoperative day, and $2.8(2.0-4.0)$ on the second postoperative day; pain scores did not differ between surgery types. Mean number of tablets used was 19 (range 0-86). Nausea occurred in 34\% of patients, more often in women ( $45 \%$ vs $19 \%$ ). Overall satisfaction was high in $73 \%$ of patients. Satisfaction was correlated with pain relief $(p<0.001)$ and inversely correlated with occurrence of nausea $(p=0.01)$.

Discussion: In this data set obtained under real-life conditions we show that the SSTS effectively managed postoperative pain in abdominal and orthopedic surgeries. Future studies should determine patient populations that benefit most from the SSTS, assess the added values versus intravenous patient-controlled analgesia, and determine the pharmacoeconomics of the system. Keywords: acute pain, opioid, postoperative pain, sufentanil, sublingual formulation

\section{Introduction}

A recent development in the treatment of moderate-to-severe postoperative pain is the introduction of a sublingual sufentanil tablet system (SSTS). ${ }^{1-6}$ The SSTS system is developed by AcelRx Pharmaceuticals (Redwood City, CA, USA) and is marketed in Europe as Zalviso ${ }^{\mathrm{TM}}$ by Grünenthal $\mathrm{GmbH}$ (Aachen, Germany). ${ }^{5}$ The noninvasive hand-held SSTS is in fact a patient-controlled analgesia (PCA) device without the need for an intravenous (IV) catheter. The SSTS device holds a cartridge with 40 microtablets, each of which contains $15 \mu \mathrm{g}$ sufentanil. The patient operates the device by placing the tip, from which the tablet is released, under the tongue while activating the system through a unique thumb tag. After activation one tablet is released to the sublingual mucosa where it is slowly absorbed. The SSTS has a lockout period of 20 minutes and the device can be used for up to 72 hours. In case of continuation of pain treatment beyond 72 hours, a new SSTS can be given to the patient or alternative analgesic regimens may be used.
Department of Anesthesiology, Leiden University Medical Centre, LUMC Albinusdreef 2, 2333 ZA Leiden, the Netherlands

Tel +3 I 7I 526230 I

Email a.dahan@lumc.nl 
The SSTS has earlier been studied in a series of registration studies. ${ }^{1-3}$ Two randomized controlled trials compared the SSTS to placebo after orthopedic (knee or hip arthroplasty) or abdominal surgery. The SSTS provided better analgesia than placebo with somewhat greater efficacy in orthopedic than abdominal surgery. ${ }^{1,2}$ In a Phase III, randomized, openlabel study, the SSTS was compared to IV PCA morphine in abdominal and orthopedic (total knee or hip replacement) surgeries. ${ }^{3}$ Significantly more patients on the SSTS than IV PCA morphine rated their pain control as good or excellent (SSTS 78.5\% vs IV PCA morphine 65.6\%, $p<0.01$ ), using the patient global assessment of method of pain control. Additionally, the SSTS was not only noninferior to IV PCA morphine in terms of analgesia but also slightly superior.

In the Netherlands, the SSTS was introduced in August 2016. Here we present the results of the first clinical experience (i.e., data obtained under real-life conditions) with the SSTS in patients following major predominantly laparoscopic-assisted abdominal procedures and orthopedic surgeries (predominantly total knee replacement surgery).

\section{Methods}

After a training phase in which both doctors and nurses were educated in its use, the SSTS was introduced in five hospitals in the Netherlands, two tertiary referral centers (Leiden University Medical Centre, Radboud University Medical Centre), and three secondary centers (Maasstad Hospital, Reinier de Graaf Hospital, and Alrijne Hospital), as an alternative to IV or oral opioid therapy in the treatment of postoperative moderate-to-severe pain. After the study was approved by the institutional review boards (identifier G16.075), the data were collected prospectively; the institutional review boards waived the requirement for written informed consent. Patient data confidentiality was maintained as all data were processed anonymously. Our initial focus was on tablet use, pain scores, prevalence of nausea, and patient satisfaction data. The SSTS was just one of the alternatives among a series of possible multimodal pain therapy options. The choice of the desired postoperative treatment (e.g., oral opioids, epidural analgesia, IV PCA with an opioid or the SSTS) is made during preoperative screening by the attending anesthesiologist together with the patient. We positioned the SSTS against standard postoperative analgesia care for major abdominal or orthopedic surgery, that is, oral opioids or IV PCA with morphine or piritramide, and all patients who previously would receive oral opioids or an IV PCA system were now given the choice of the SSTS. Exclusion criteria were 1) age $<18$ years; 2) inability to understand the instructions; 3 ) inability to operate the device;
4) oral cavity surgery; 5) postoperative epidural analgesia; 6) known allergy to sufentanil. In one center, Maasstad Hospital, the SSTS was introduced as standard treatment for all patients after knee arthroplasty.

Most patients received general anesthesia with propofol induction followed by maintenance with either sevoflurane or propofol; most patients undergoing orthopedic procedures received spinal anesthesia. After surgery, all patients after general anesthesia were titrated to pain scores $<4$ (measured on an 11-point numerical rating scale ranging from 0 , no pain, to 10 , most severe pain imaginable) before the treatment with the SSTS was started. Titration was according to local protocol and involved dose titration with morphine or piritramide. Patients after spinal anesthesia received the SSTS in the post-anesthesia care unit (PACU) and were instructed to use the system when they felt pain and (subjectively) felt the need for pain relief. Apart from the SSTS all patients received paracetamol and a non-steroidal anti-inflammatory drug (NSAID) three times per day as per local protocol. Antiemetic therapy (ondansetron, dehydrobenzoperidol, and dexamethasone) was given upon occurrence of nausea or was given preemptively in patients with known risk factors for nausea (Apfel score). This was done according to local protocol.

The following data were collected: patient age, weight, and gender, type of surgery, tablet use (total number of tablets used and tablet use in the first 24 hours), duration of SSTS use, pain scores during SSTS treatment (mean daily numerical rating scores at rest [NRS-rest] derived from three measurements per 24 hours), concomitant pain medication apart from paracetamol or an NSAID, occurrence of nausea, and patient satisfaction score (scored once when the SSTS treatment was terminated, on a three-point qualitative scale: low, moderate, and high satisfaction). Data collection ended at the end of SSTS treatment. NRS values are presented as median \pm interquartile range (IQR) to represent the general pain score during SSTS treatment. All other data are presented as mean (SD) or percentages.

Although this is an exploratory representation of a single treatment cohort we performed several pragmatic analyses. A multivariate analysis was performed to assess the influence of NRS, the presence of nausea, center, anesthesia type, and surgery type on patient-reported satisfaction scores. Gender effects on tablet consumption and NRS were analyzed using the Mann-Whitney test. As abdominal and orthopedic surgeries were most prevalent in our cohort with a substantial number of patients (186 and 95 patients for abdominal and orthopedic procedures, respectively), we compared the two groups with respect to NRS, tablets used, and satisfaction scores. The data 
were analyzed using GraphPad Prism version 7 for MAC OS X, GraphPad Software, La Jolla, CA, USA, or SPSS (IBM Corp., IBM SPSS Statistics for Windows, version 22.0. Armonk, NY, USA). $P$-values $<0.05$ were considered significant.

\section{Results}

Three hundred patients were recruited in the study; we report on the data of 280 patients, as data acquisition was incomplete in 20 patients. Patient characteristics and study observations are summarized in Table 1 . The majority of patients

Table I Characteristics of Zalviso ${ }^{\mathrm{TM}}$ users and study observations

\begin{tabular}{|c|c|}
\hline Variable & Value \\
\hline Number of patients included in the study & 280 \\
\hline Men/women (n/n) & $116 / 164$ \\
\hline Age (years, median, range) & $61(18-86)$ \\
\hline Weight (kg, mean $\pm \mathrm{SD}$, range) & $83.9 \pm 18(50-140)$ \\
\hline Intra-abdominal surgery (n) & 137 \\
\hline Orthopedic surgery (n) & 95 \\
\hline Miscellaneous procedures (n) & 48 \\
\hline Duration of Zalviso use (hours, mean $\pm S D$, range) & $43 \pm 21(1-160)$ \\
\hline Total tablets used (mean $\pm S D$, range) & $19 \pm 14(0-86)$ \\
\hline Tablets per hour (mean $\pm S D$, range) & $0.48 \pm 0.4 \mathrm{I}(0-2.0)$ \\
\hline $\begin{array}{l}\text { Tablets used in the first } 24 \text { hours (mean } \pm \text { SD, } \\
\text { range) }\end{array}$ & $12 \pm 9(0-48)$ \\
\hline $\begin{array}{l}\text { Tablets per hour in the first } 24 \text { hours (mean } \pm S D \text {, } \\
\text { range) }\end{array}$ & $0.49 \pm 0.39(0-2.1)$ \\
\hline NRS-rest on the day of surgery (median, IQR) & $3.5(2.3-4.0)$ \\
\hline NRS-rest on first day after surgery (median, IQR) & $3.2(2.3-4.0)$ \\
\hline $\begin{array}{l}\text { NRS-rest on second day after surgery (median, } \\
\text { IQR) }\end{array}$ & $2.8(2.0-4.0)$ \\
\hline Prevalence of nausea (total population) & $34 \%$ \\
\hline Nausea in men & $19 \%$ \\
\hline Nausea in women & $45 \%$ \\
\hline \multicolumn{2}{|l|}{ Satisfaction with pain treatment } \\
\hline Low satisfaction & $12 \%$ \\
\hline Moderate satisfaction & $15 \%$ \\
\hline High satisfaction & $72 \%$ \\
\hline \multicolumn{2}{|l|}{ Satisfaction in patients with an NRS $>4$} \\
\hline Low satisfaction & $44 \%$ \\
\hline Moderate satisfaction & $19 \%$ \\
\hline High satisfaction & $36 \%$ \\
\hline \multicolumn{2}{|l|}{ Satisfaction in patients without nausea } \\
\hline Low satisfaction & $8 \%$ \\
\hline Moderate satisfaction & $14 \%$ \\
\hline High satisfaction & $78 \%$ \\
\hline \multicolumn{2}{|l|}{ Satisfaction in patients with nausea } \\
\hline Low satisfaction & $20 \%$ \\
\hline Moderate satisfaction & $21 \%$ \\
\hline High satisfaction & $58 \%$ \\
\hline \multicolumn{2}{|l|}{ Satisfaction in patients with nausea and NRS $>4$} \\
\hline Low satisfaction & $45 \%$ \\
\hline Moderate satisfaction & $22 \%$ \\
\hline High satisfaction & $33 \%$ \\
\hline
\end{tabular}

Notes: Zalviso ${ }^{T M}$ manufacturered by Grunenthal GmbH (Aachen, Germany) Abbreviations: IQR, interquartile range; NRS, numerical rating score. underwent abdominal surgery (49\%), of which $70 \%$ was performed laparoscopically (colon or rectum resection). The rest was either orthopedic (34\%, knee replacement surgery) or other surgery $(17 \%)$, including mastectomy, vascular surgery, plastic surgery, thoracic surgery, or hernia correction.

The average number of consumed tablets was 18.6 (14.4) with a range of $0-86$, corresponding to 279 (216) $\mu \mathrm{g}$ and range $0-1290 \mu \mathrm{g}$. The majority of tablets was consumed in the first 24 hours after surgery (13.3 [9.4], range 0-48; 199.5 [141] $\mu \mathrm{g}$, range $0-720 \mu \mathrm{g})$. Eight patients did not make use of the system, and 88 patients used fewer than 10 tablets. Eight patients used more than one cartridge of 40 tablets. The duration of the SSTS was on average 43.2 (21) hours, with one outlier, a patient who used the system for 160 hours. Most patients used the SSTS for 24, 48, or 72 hours. There was no difference in tablet consumption between men and women (19.7 [14.6] vs 18.4 [14.2] tablets; 295.5 [219] vs 276 [213] $\mu \mathrm{g})$. No correlation was found between weight and tablet consumption $(p=0.75)$. On average, patients used 0.48 (0.41) tablets per hour (7.2 [6.2] $\mu \mathrm{g}$ per hour) or one tablet $(15 \mu \mathrm{g})$ every 2 hours. There was a small age effect with elderly patients using less tablets (reduced tablet use is 0.2 tablet per hour, $p=0.02$ ).

As shown in Figure 1, median pain scores (IQR) on postoperative days 0,1 , and 2 during STTS treatment were 3.5 (2.3-4.0), $3.2(2.3-4.0)$, and 2.8 (2.0-4.0). Irrespective

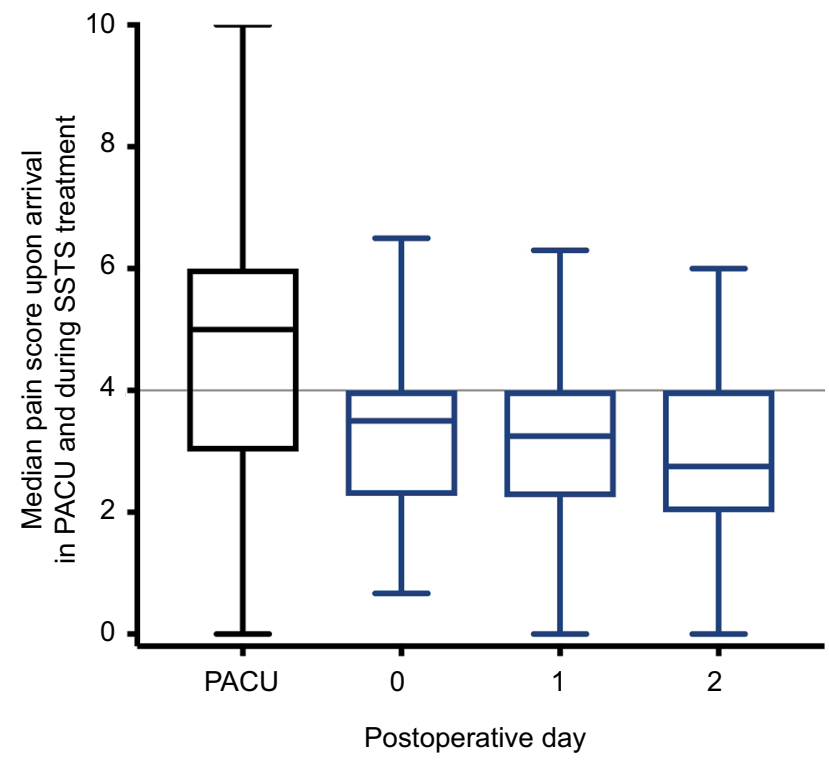

Figure I Box plots of pain scores upon arrival in the post-anesthesia care unit (only the data from patients after general anesthesia were used), and pain scores of all patients observed during day 0 (day of surgery) and days $I$ and 2 after surgery. Note: Pain scores were obtained three times per day and the daily averages were used for analysis.

Abbreviation: PACU, post-anesthesia care unit; SSTS, sublingual sufentanil tablet system. 
of the treatment day $25 \%$ of patients reported an NRS-rest $>4$. NRS-rest was not correlated to patient weight. During the course of treatment, 28 patients $(10 \%)$ had insufficient pain relief from the SSTS and required either rescue medication (e.g., IV methadone, IV esketamine, clonidine, tramadol, oxycodone) or were switched to IV PCA morphine, oral oxycodone, or subcutaneous morphine. Nausea occurred in $34 \%$ of patients, $19 \%$ of men and $45 \%$ of women, pruritis occurred in five patients, sedation in four patients, delirium in four elderly patients, and respiratory depression in one patient (as detected by hypopnea and an increase in arterial partial pressure of carbon dioxide).

Twelve percent of patients reported low satisfaction, 15\% moderate satisfaction, and 73\% high satisfaction (Figure 2). Multivariate analysis indicated that satisfaction correlated to NRS at rest $(p<0.001)$ and the presence of nausea $(p=0.01)$. Neither satisfaction with pain relief nor the influence of pain or nausea on satisfaction with pain relief was related to gender, type of surgery, type of anesthesia, hospital, or age. Median NRS values at low, moderate, and high satisfaction were 4.1 (2.8-5.2), 2.9 (2.3-4.0), and 2.7 (2.0-3.5), respectively.

Compared to orthopedic surgery, NRS-rest was slightly lower after abdominal surgery (2.8 [1.4] vs 3.2 [1.2], $p=0.01)$. However, total tablet use was less in orthopedic patients (15.1 [11.0] vs 19.5 [14.4], $p<0.01$ ). There was no statistically significant differences in duration of SSTS use between abdominal and orthopedic surgeries (46 \pm 23 hours vs $41 \pm 15$ hours, $p=0.10$ ). Satisfaction scores were similar with high scores in 74\% (abdominal patients) and 72\% (orthopedic patients; Figure 2).

In Box 1 we report a collection of observed problems with the use of the system. Problems were diverse and were specifically related to tablets (e.g., problems with absorption), thumb tag, or the hand-held device (e.g., problems with operation). One patient shared one tablet with her roommate after she had activated the system herself.

\section{Discussion}

Our case series of 280 patients indicates that postoperative pain management with sublingual sufentanil is effective following a variety of surgical interventions. As part of a multimodal approach, sublingual sufentanil was effective in $90 \%$ of patients. In $75 \%$ of patients pain scores were below 4 . Satisfaction scores were high in $73 \%$ of patients, irrespective of gender or surgery type. Reduced satisfaction scores were related to the incidence of nausea and high pain scores. In $10 \%$ of patients rescue medication was required or treatment was considered ineffective and changed to an alternative analgesic regimen. In our experience this is similar to other postoperative pain treatments such as IV PCA morphine or even epidural analgesia. The incidence of nausea in $34 \%$ of patients and the higher prevalence in women is not unexpected as sufentanil will evidently not be exempt of opioidrelated side effects. Earlier, we observed nausea in $52 \%$ of patients on IV PCA morphine, again with a higher occurrence

Box I Issues with the use of the sublingual sufentanil tablet system Issues with the tablets

- Problems with tablet absorption because of dry mouth $(n=4)$

- Multiple tablets retained in the mouth because of dry mouth $(n=I)$

- Tablets found in bed $(n=3)$

Issues with the thumb tag

- The thumb tag had to be replaced (did not function/got off/got lost) $(n=22)$

- The thumb tag was swallowed $(n=l)$

- The thumb tag prevents smartphone use $(n=l)$

Issues with the hand-held device

- Difficulty with finding the mouth and/or lifting the tongue $(n=2)$

- Cap on device tip not removed by the patient $(n=4)$

- Device tethered to the bed got lost when patient switched beds $(n=I)$

- Device failure (no tablet released) $(\mathrm{n}=30)^{\mathrm{a}}$

Miscellaneous issues

- Patient shares tablet with other patients $(\mathrm{n}=\mathrm{I})$

Notes: ${ }^{\text {AAll }}$ patients received a second system that was operational. This issue has been resolved by the manufacturer.
A

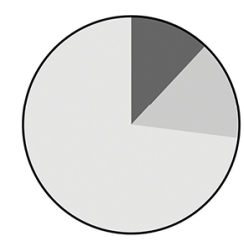

All patients
B

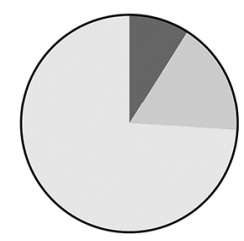

Intra-abdominal surgery
C

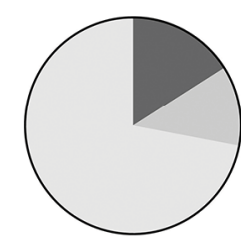

$\square$ Low satisfaction Moderate satisfaction $\square$ High satisfaction

Orthopedic surgery

Figure 2 Pie charts of satisfaction scores of patients treated with the sublingual sufentanil tablet system.

Notes: Panel (A) depicts all patients, panel (B) patients following abdominal surgery, and panel (C) patients following orthopedic surgery. 
in women than men. ${ }^{7}$ In the current study we did not collect data on antiemetic therapy. Furthermore, we did not take into account the use of opioids during anesthesia. Hence, future studies will have to ascertain whether sublingual sufentanil per se causes less nausea than other postoperative opioids. Additionally, it is important to realize that the choice for using the SSTS in this observational study was actively made by the anesthesiologist (together with the patient). This may have caused attribution bias. Still, the sample of patients presented in this report was very similar to those of our regular clinical practice. Furthermore, all patients in one center after knee arthroplasty were included.

The high satisfaction scores observed in the study of Melson et $\mathrm{al}^{3}$ are in close agreement with our observation $(78 \%$ vs $73 \%)$. These high satisfaction scores might be related to the relatively rapid onset of pain relief with sublingual sufentanil and the relatively low incidence of nausea. In contrast to the registration studies, we did not find a large difference in efficacy between orthopedic and abdominal procedures.

We found certain issues with the operation of the device (Box 1). For some, especially elderly patients, using the system was difficult and sometimes assistance was required. It is evident that the device is unsuitable for cognitively impaired patients (as is also true for IV PCA morphine), patients with a delirium, patients with coordination problems, patients who cannot hold the device in their hand (e.g., patients with severe rheumatoid arthritis), or patients after oral cavity surgery. In a few patients the dryness of the oral mucosa prevented sufficient absorption of the tablet. Most of the problems, however, are inherent to the introduction of a relatively new technique with a learning curve for patients, doctors, and nurses. It is our experience that simply informing the patient how to operate the device is insufficient. In addition, several issues need to be considered (Box 1). Equally important, health professionals will need to determine the suitability of each patient for the SSTS. In our experience, the location and timing to assess suitability are not limited to the preoperative screening clinic but suitability should be reassessed on the ward (or PACU) just prior to initiation of treatment. Evidently, postoperative patient conditions may be such that operation of the device is now more difficult than earlier anticipated.

The pharmacoeconomics of the system is an important issue. The current market price of the SSTS device in the Netherlands is $€ 895$ (US $\$ 1,100$, BP £880) while the market price of the consumables is $€ 100$ (US\$125, BP £90) per patient, depending on the number of consumables purchased (listing of February 2018). Not all patients included in our study were suitable for the SSTS treatment option. A substantial number of patients $(31 \%)$ used $<10$ tablets during the course of the postoperative pain treatment, with 15 patients using either no or just one tablet. Often this one tablet was a test tablet that was given to the patient by the nursing staff during the start of treatment. Patients who use only a few tablets are possibly better off with an alternative analgesic regimen, such as on-demand oral tramadol or low-dose oxycodone. Further experience with the system is needed to assess which patient population may benefit the most from the SSTS.

We observed no correlation between weight and tablet use or weight and NRS. This is an important observation and suggests that the fixed sufentanil dose serves a large variety of weights. The weight of our patients ranged from 50 to 140 $\mathrm{kg}$ with $25 \%$ of patients with a weight above $90 \mathrm{~kg}$ and $25 \%$ with a weight below $70 \mathrm{~kg}$. One has to be careful in using the SSTS in patients with weights below $50 \mathrm{~kg}$ as this may cause more intense opioid side effects. Similarly, pain relief from the SSTS in patients with weights above $140 \mathrm{~kg}$ may possibly be inadequate.

In conclusion, the sufentanil sublingual tablet system was without major complications in our cohort and is a viable alternative to IV PCA in postoperative patients without the need for an IV access. Future studies should 1) determine the patient populations that benefit most from the SSTS; 2) extend the comparison with IV PCA morphine in other surgical models; 3) compare the SSTS with other non-IV forms of analgesia, including epidural analgesia; and 4) perform an appropriate pharmacoeconomic evaluation of this new treatment option. As item 4 is highly country-specific, separate analyses per country are needed.

\section{Disclosure}

Prof. Dahan received speaker/consultancy fees from AcelRx and Grünenthal. The authors report no other conflicts of interest in this work.

\section{References}

1. Jove M, Griffin DW, Minkowitz HS, Ben-David B, Evashenk MA, Palmer PP. Sufentanil sublingual tablet system for the management of postoperative pain after knee and hip surgery. Anesthesiology. 2015;123(2):434-443.

2. Ringold FG, Minkowitz HS, Gan TJ, et al. Sufentanil sublingual tablet system for the management of postoperative pain following open abdominal surgery: a randomized, placebo-controlled study. Reg Anesth Pain Med. 2015;40(1):22-30. 
3. Melson TI, Boyer DL, Minkowitz HS, et al. Sufentanil sublingual tablet system vs. intravenous patient-controlled analgesia with morphine for postoperative pain control: a randomized, active-comparator trial. Pain Pract. 2014;14(8):679-688.

4. Willsie SK, Evashenk MA, Hamel LG, Hwang SS, Chiang YK, Palmer PP. Pharmacokinetic properties of single- and repeated-dose sufentanil sublingual tablets in healthy volunteers. Clin Ther. 2015;37(1):145-155.

5. van de Donk T, Ward S, Langford R, Dahan A. Pharmacokinetics and pharmacodynamics of sublingual sufentanil for postoperative pain treatment. Anaesthesia. 2018;73(2):231-237.
6. Katz P, Takyar S, Palmer P, Liedgens H. Sublingual, transdermal and intravenous patient-controlled analgesia for acute post-operative pain: systematic literature review and mixed treatment comparison. Curr Med Res Opin. 2017;33(5):899-910.

7. Binning AR, Przesmycki K, Sowinski P, et al. A randomised controlled trial on the efficacy and side-effect profile (nausea/vomiting/ sedation) of morphine-6-glucuronide versus morphine for post-operative pain relief after major abdominal surgery. Eur J Pain. 2011;15(4): 402-408

\section{Publish your work in this journal}

The Journal of Pain Research is an international, peer reviewed, open access, online journal that welcomes laboratory and clinical findings in the fields of pain research and the prevention and management of pain. Original research, reviews, symposium reports, hypothesis formation and commentaries are all considered for publication.
Dovepress

The manuscript management system is completely online and includes a very quick and fair peer-review system, which is all easy to use. Visit http://www.dovepress.com/testimonials.php to read real quotes from published authors. 